\title{
Some remarks on experimental estimation of damping for seismic design of civil constructions
}

\author{
Carlo Rainieri ${ }^{\mathrm{a}, *}$, Giovanni Fabbrocino ${ }^{\mathrm{a}}$ and Edoardo Cosenza ${ }^{\mathrm{b}}$ \\ ${ }^{a}$ Structural and Geotechnical Dynamics Lab StreGa, Department SAVA, University of Molise, School of \\ Engineering, Via Duca degli Abruzzi, 86039 Termoli, Italy \\ ${ }^{\mathrm{b}}$ Department of Structural Engineering, University of Naples “Federico II”, Via Claudio 21, 80125 Naples, Italy
}

\begin{abstract}
Experimental estimation of damping is currently not a comprehensively solved problem. Although modal frequencies and mode shapes can be measured confidently and quite easily by means of dynamic tests on civil structures, an accurate identification of damping ratios needs further development. Experimental values can be characterized by large error bounds mainly because damping is strongly influenced by the magnitude of the dynamic response of a structure.

In this paper, the main issues concerning identification of modal damping ratios are discussed in order to define methods for their reliable estimation, to reduce uncertainties and characterize error bounds. According to this aim, a procedure for optimized modal parameter estimation via Stochastic Subspace Identification is also proposed. Then data collected from an extensive literature review are analyzed depending on the type of structure in order to point out the main factors affecting damping. Moreover, a comparison with provisions about structural damping given in National and International seismic codes for structural design of civil structures is reported. This process demonstrates that the development of a damping database based on homogeneous and reliable estimates is required for calibration of predictive formulations to be used in the framework of performance-based seismic design.
\end{abstract}

Keywords: Dynamic tests, damping estimation, operational modal analysis, performance-based design

\section{Introduction}

The fundamental law of motion governing structural dynamics has been deeply investigated by many researchers, in particular to take into account the effect of damping on the dynamic behaviour of structures. Nevertheless, despite of the large amount of literature available on the subject, damping is a crucial aspect of general vibration analysis. In fact, a single well established formulation to represent damping forces does not exist [1]. However, damping strongly influences the structural response under dynamic loads. This circumstance affects both the ultimate limit state and the serviceability limit state of civil constructions.

Horizontal dynamic forces like wind and seismic excitation due to ground motion are key issues for structural design. In case of tall buildings, for instance, wind-induced accelerations are of primary importance for resident comfort concerns and they can be reduced by increasing structural damping. Thus, since vibration levels in serviceability conditions have to be taken into account for an effective design, an increasing attention has been recently focused on damping evaluation for the purpose of designing vibration control devices. On the other hand, since dynamic displacements, strains and stresses in a structure subjected to seismic excitation are strongly influenced by damping, its accurate estimation is crucial also for a proper seismic design of structures.

\footnotetext{
*Corresponding author. E-mail: carlo.rainieri@unimol.it.
} 
Although natural frequencies and mode shapes can be accurately measured with moderate complexity by dynamic tests, damping estimation still represents a problem, and error bounds in experimental values can be large. On the other hand, mathematical models may predict natural frequencies and mode shapes of a structure. Conversely, an extensive computational effort is generally required for analytical estimation of damping. A comprehensive description of damping and how it can be modelled by Finite Element approach can be found in [2]. Interesting results have been obtained by some researchers in this field [3,4]; however, applications are basically related to structures, such as beams or assemblies with bolted joints, which are of interest in mechanical engineering but they are not representative of the structural complexity of civil constructions. The latter are, in fact, characterized not only by hysteretic damping or by dissipations due to friction in joints. The energy dissipation is, in fact, the result of interaction among several different damping mechanisms, both internal (related, for instance, to material) and external (due, for instance, to the interaction between structural and non-structural members). Moreover, the values commonly adopted for dynamic analyses are basically empirical values, based on experimental estimates of damping obtained from similar structures. However, it is worth noting that structures are often only superficially similar, since they could be affected by substantial differences, for example, in arrangement, dimensions and use of materials and structural details.

When response spectrum modal analyses are carried out, under the assumption of proportional damping the response of the structure is obtained as the superposition of responses of independent viscously damped Single Degree Of Freedom (SDOF) systems. In such a case, response amplitudes are inversely proportional to damping coefficients. Thus, the use of low damping values is conservative but it can result in an overdesigned structure. On the other hand, if the damping value used for the analyses is overestimated, actual stresses in the structure subjected to dynamic loads are underestimated. It is, therefore, of primary importance the availability of a reliable prior estimate of the damping capacity of a structure, in order to achieve both a safe and economical design of the structure itself.

This target can be accomplished by creating a large database of experimentally obtained damping values, so that correlations can be found, allowing estimation of damping values for different structural typologies and levels of vibration according to an empirical base. It is, therefore, necessary to define homogeneous classes of structures and the corresponding expected values for damping, and reduce error bounds of experimental estimates by adopting appropriate procedures for damping extraction.

In this context, the main contribution of the present paper is related to the definition of likely criteria for a reliable damping estimation, within the limitations of the estimators, by reducing the scatter due to improper data processing; moreover, a literature review has been carried out in order to define the main factors influencing damping and some typical values for different typologies of structures. It is worth emphasizing that collected data are not exhaustive to define any correlation, not only due to their quantity, but also for the lack of detailed information on test structures and data processing, thus only average values and the corresponding standard deviations computed from available data are reported for some classes of building-like structures.

\section{Damping mechanisms}

Several damping mechanisms can be found on a certain structure. They can be generally classified as [5]:

- Damping intrinsic to the structural material;

- Damping due to friction in the structural joints and between structural and non-structural elements;

- Energy dissipated in the foundation soil;

- Aerodynamic damping;

- Damping introduced by passive and active dissipation systems.

The function of damping is to dissipate energy and limit the magnitude of forced vibrations in a structure: in this sense, the specific damping capacity of a structure can be defined as the percentage of the total energy of vibration lost in a cycle, that is provided by the ratio between the energy lost in a cycle and the corresponding maximum stored energy.

The different damping mechanisms acting on a structure are usually represented by a unique model, the equivalent viscous damping model, which takes into account all dissipation sources in a construction. However, a brief 
discussion about the different damping mechanisms is herein reported in order to better understand the behaviour of equivalent viscous damping, sometimes called also structural damping, and the main factors influencing its magnitude.

Structural damping usually consists of one or a combination of the following types of damping: viscous damping, hysteretic damping, friction (or Coulomb) damping, aerodynamic (or atmospheric) damping.

Viscous damping is assumed to be proportional to the velocity of the oscillatory motion. In such a case, the vibratory motion of the SDOF system is described by the following differential equation:

$$
m \ddot{x}(t)+c \dot{x}(t)+k x(t)=F(t)
$$

where $m$ is the mass, $c$ the viscous damping and $k$ the stiffness of the SDOF system; $F$ is the dynamic force acting on the system; $\ddot{x}, \dot{x}$ and $x$ denote the system response in terms of acceleration, velocity and displacement, respectively.

When $c$ is above the critical value $c_{c r}$ :

$$
c_{c r}=2 \sqrt{k m}
$$

that is to say, the damping ratio:

$$
\zeta=\frac{c}{c_{c r}}=\frac{c}{2 \sqrt{k m}}
$$

is larger than 1 , the initially disturbed system will not oscillate but it will simply return to the equilibrium position. It can be also defined as the smallest amount of damping for which no oscillations occur in the free response [6]. This condition does not commonly occur in practice [7].

Real structures usually show a damping much lower than the critical damping (damping is generally lower than $10 \%$ of critical value). In these cases, the frequency of vibration of a system (its damped frequency) is basically equal to the (undamped) natural frequency [8]. For an underdamped system, motion is oscillatory and characterized by an amplitude of vibration which decreases exponentially.

The hysteretic damping is described by the following frequency domain model $[9,10]$ :

$$
-m \bar{\omega}^{2} \bar{X}+k(1+\operatorname{sgn}(\bar{\omega}) j \eta) \bar{X}=\bar{P}
$$

where $j=\sqrt{-1}, \eta$ is the loss factor, $k \eta=h$ represents the hysteretic damping coefficient and $\operatorname{sgn}$ is the signum function.

Based on the Inverse Fourier Transform of Eq. (4), the governing equation in time domain, as derived by Chen [11] and Inaudi and Kelly [12] independently, can be written as follows:

$$
m \ddot{x}(t)-\frac{k \eta}{\pi} \int_{-\infty}^{+\infty} \frac{x(\tau)}{(t-\tau)} d \tau+k x(t)=p(t)
$$

with the following conditions:

$$
\left.x(t)\right|_{t=-\infty}=0 ;\left.\dot{x}(t)\right|_{t=-\infty}=0
$$

The integral-differential Eq. (5) in time domain is equivalent to the complex stiffness model (4) in frequency domain, being them related through a Fourier Transform. It is worth noting that this hysteretic damping model violates the requirement of causality, that is to say the system responds before being excited (see [13-16] for additional details).

Hysteresis is basically due to inelastic behaviour: thus an equivalent damping ratio can be obtained from the area sketched by the hysteresis loop.

Material damping is another example of hysteretic damping. For concrete or soils [17], damping inherent to material may be relevant ( 0.05 is the typical value for concrete); for structural metals, instead, the damping capacity is much smaller.

A SDOF system characterized by friction damping is described by the following differential equation:

$$
m \ddot{x}(t)+k x(t)+R x(t) /|x(t)|=F(t)
$$

where $R=\mu N$ is the friction force, $\mu$ takes into account the coefficients of static and kinetic friction and $N$ is the normal force between the sliding surfaces. Friction damping is, therefore, the result of rubbing and sliding between 
vibrating dry surfaces. It is proportional to amplitude and changes its sign according to the sign of motion. A freely vibrating system subjected to pure friction damping shows a linearly decaying amplitude.

Much of the energy dissipated in vibrating structures is due to friction. However, a structure usually exhibits at least a combination of hysteretic and friction damping. This kind of combination is pointed out by an initial increase and a subsequent decrease of damping capacity with increasing amplitude of motion [17].

Aerodynamic damping arises as a result of the drag and lift forces on an object in air. Such forces are proportional to the square of the velocity of the object with respect to the air stream.

In technical literature a number of relationships for aerodynamic damping prediction can be found [17]. Even if aerodynamic damping is much lower than other types of damping mechanisms, it can give a relevant contribution to the overall damping for some specific types of structures, i.e. tall buildings or long span bridges.

Real dynamic systems show, in general, a combination of linear (that is to say, damping independent of amplitude of motion) and non-linear (namely, damping depending on amplitude) damping mechanisms. As a consequence, there is not a single way to describe mathematically all vibrating structures. In engineering practice, due to the difficulty of defining the true damping characteristics of typical structural systems, the equivalent viscous damping model is usually used, because it leads to linear equations of motion. Even when viscous damping may be not operating, such model is generally assumed, and a distinction between existence of pure viscous damping or assumption of equivalent viscous damping is rarely found in the literature.

In most design codes, damping is usually assigned depending on the selected construction material. For example, the Italian Seismic Code [18] gives a spectrum computed for a damping value equal to $5 \%$ and suggests a formulation to modify the spectrum when the actual damping is different. However, no specific suggestions are given to define the design value for damping; it is only pointed out that its evaluation has to be based on construction materials, structural typology and type of foundation soil.

This applies to design methods based on the comparison between forces acting on the structures (Force Based Design - FBD) and also to design procedures based on the comparison between displacements (Displacement Based Design - DBD) [19]. In all cases, design checks require that, in probabilistic terms, the seismic demand D, depending on the dynamic response of the structure, is lower than the seismic capacity $\mathrm{C}$, depending on the local and global ductility of constructions.

Seismic demand is correlated to damping offered by the structures at the limit state of interest, either serviceability or ultimate. The former provides a threshold for the structural performance of the construction under frequent earthquakes and it is aimed at prevent damage of non-structural components and systems by limiting structural displacements. The latter represents the threshold value beyond which it is assumed that the remaining capability of the structure to bear both horizontal and vertical forces is very low due to extent and relevance of structural damage.

It is worth noting that according to FBD, damping affects design spectra and, therefore, it controls the forces acting on the system; conversely, when DBD is concerned, an equivalent damping ratio has to be set depending on the main characteristics of the structure and the inelastic range induced by ground motion.

In this context, the value of 0.05 for damping seems to fulfil requirements for design at ultimate limit state of reinforced concrete structures, when they are required to behave in a strongly non-linear manner due to ground motion and material (hysteretic) damping becomes the predominant dissipation mechanism. Conversely, for different structural typologies or for frequent earthquakes (characterized by low or moderate levels of ground shaking, for which the structure is designed so that it should behave linearly), this value can lead to a substantial underestimation of the structural response.

For wind applications, the ISO Code [20] suggests damping ratios of $1 \%$ and $1.5 \%$ for steel and reinforced concrete structures, respectively. It is worth noting that damping exhibits an increase with amplitude in full-scale data sets. This circumstance may be explained by taking into account that the equivalent viscous damping model is usually adopted in the equations of motion to represent all the different damping mechanisms. However, this model is nonideal under a wide range of amplitudes of motion and, if it is assumed, actual structures may exhibit non-linearity, or a damping that changes with amplitude. Thus, a model for total damping with respect to amplitude of motion has been proposed (see, for example [21]). Assuming that frictional losses give a large contribution to energy dissipation in structures, at low levels of motion there are no significant slipping phenomena in the structure. Once a sufficient number of interfaces are activated, they will dissipate energy in proportion to their relative displacements, and it is possible to consider a linear increase of damping. At high amplitudes of motion, all interfaces have been activated 
and friction forces become constant even for increasing amplitude, thus defining the maximum level of damping that should not be exceeded unless damage occurs within the structure. In fact, in large amplitude regime, damping ratios can increase only if there are additional sources of damping forces: there is the possibility of damage to secondary elements, but design codes usually do not allow this kind of damage, at least for certain levels of excitation. Thus, in such cases, higher damping ratios cannot be expected for structures vibrating within their elastic limit. If, instead, the amplitude exceeds this limit, the contribution of plastic-hysteretic damping may become predominant.

The non-linear model of damping behaviour with vibration amplitude has been justified, from a theoretical standpoint, according to the principles of fracture mechanics in [22] and an autoregressive model for damping prediction in buildings has been proposed in [23]. Even if some refinements are still needed, the latter could represent a promising tool for estimation of damping at high levels of vibration starting from damping estimated at low amplitudes of motion (such as damping estimates in operational conditions). Thus it can fill the lack of damping data for structures strongly shaken but within their elastic range and improve design of structures for moderate earthquakes.

\section{Experimental damping estimation}

Estimation of damping can be carried out using measurements of the structural response to forced, transient or ambient excitation. In [24] several techniques requiring the knowledge of the input force are described. Such techniques are extensively applied in laboratory testing in the field of aerospace and mechanical engineering.

The most popular procedures are based on the FRF curve-fit or on the Nyquist diagram circle-fit. These curve fitting methods probably provide the most accurate estimation of damping for a given structure.

However, they cannot be easily applied to large civil structures such as tall buildings, bridges or dams, due to the need of artificially induced vibrations large enough to overcome the ambient noise, but not too large in order to avoid non-linearities. Other methods described in [24] and working in time domain are, instead, the Ibrahim Time Domain technique and methods based on auto-regressive models.

When forced or transient vibration tests are used for damping estimation, shakers or instrumented hammers are used in order to excite the structure. Sometimes, snap-back techniques are used, by elastically deforming the structure and suddenly releasing it in order to measure damping from free decay oscillations. However, when input is applied by a hammer, the transient response is the sum of the response of several modes of vibration; when snap-back techniques are applied, instead, response is mainly due to the mode (usually the fundamental mode of the structure) whose shape best resembles the statically deformed configuration of the structure.

If a shaker is used, a run-down test can be carried out. Once the resonant frequency is identified and a steady-state of forced vibration is reached, the shaker is switched off and the resulting decaying vibrations are measured. In such a case, damping is obtained applying the logarithmic decrement method. Whenever response spectrum is available, the half-power bandwidth method can be applied. However, it requires well-separated modes and it has been proved that it can lead to inaccurate damping estimates, in particular in the case of ambient vibration tests [17]. Curve fitting techniques seem to be more reliable with respect to the problem of damping estimation, since all available points are used for the fit instead of only three points like in the half power bandwidth method [17].

Recent improvements in data processing and measurement hardware performance have made forced vibration less popular than ambient vibration testing in the case of large civil structures, also because their excitation at very low frequencies is impractical or impossible with shakers, being the first natural frequencies of such structures well below the operating range of the exciter. When ambient vibration tests are used for damping estimation, since there is no control over the input force, there is also a lack of knowledge about the spectral distribution of its energy.

The options are to use time domain modelling techniques, or to determine the frequency content of response data and use frequency domain techniques, such as half-power bandwidth or curve fitting. However, the assumptions about the input in ambient vibration tests are not always satisfied and, in any case, it is impossible to judge their validity. Thus, damping estimates are affected by errors not only due to the noise in the signal and, eventually, to the errors caused by windowing in spectral analysis, but also due to the erroneous assumptions about input. Measurement noise affects the quality of fit in curve fitting procedures and, as a consequence, reliability of damping estimates. 
The use of the Hanning window in order to reduce leakage effects in spectral analysis of ambient vibration records, instead, yields a bias error with respect to the true damping value. In order to minimize this effect, a fine frequency spacing must be adopted. In order to have a good estimation of response spectra, a high number of averages is also required [25].

It has been shown [17] that, by increasing the number of averages, the damping estimates converge to a value depending on the type of curve fit, but if fewer than 10-15 averages are used, the damping estimates are significantly lower than this converged value. Frequency resolution, instead, affects the number of data points used for the fit. In [17] it is suggested to use at least 16 points in the fit, based on the results of a number of numerical simulations. As a consequence, a reliable damping estimation in the frequency domain cannot leave out of consideration long records of the structural response, in order to compute spectra characterized by a high number of averages and a fine frequency resolution. A total record length equal to 1000-2000 times the first natural period of the tested structure is usually suggested.

In [25] a simplified formulation to compute the total record length on the base of the expected first natural frequency and damping ratio is proposed. It is defined so that a high number of averages in spectrum computation is obtained. About the bias error introduced by windowing, a procedure [17] to correct damping estimates has been proposed, but it requires calibration.

In the framework of ambient vibration tests, Enhanced Frequency Domain Decomposition (EFDD) [26,27] and Stochastic Subspace Identification (SSI) [28,29] are widely used techniques for modal parameter identification. However, in order to obtain reliable estimates, in particular of damping ratios, some basic test and data processing rules should be defined. Being the EFDD method based on computation of spectra from recorded data, long records are required to keep low the error on spectrum estimation [30] and, therefore, to extract modal parameters in a reliable way. As previously mentioned, a high number of averages and a fine frequency resolution are crucial for an effective estimation of damping ratios, apart from the bias introduced by windowing. The effect of frequency resolution on modal damping ratio estimates, when the EFDD procedure is applied, has been extensively studied in [31]. It has been shown that estimated damping ratios for all identified modes decrease when the frequency resolution improves. In particular, they have found that damping estimates converge for a frequency resolution equal to $0.01 \mathrm{~Hz}$ or better. Moreover, the bias in damping estimation is kept low by inverse Fourier transforming the identified SDOF Bell functions and by fitting the data related only to the first few cycles of the obtained correlation functions for the identified modes.

Even if the EFDD technique is, in principle, able to deal with closely spaced modes, damping estimation in such a case seems to be not very accurate. Partial identification of SDOF Bell functions, beating phenomena and errors due to windowing can significantly bias damping estimates. A more refined identification of damping ratios in presence of close modes can be obtained by means of SSI methods. This ability is crucial above all for very flexible structures, characterized by low natural frequencies and damping ratios and by several close modes.

SSI methods are used to provide estimates of modal parameters also in presence of limited amounts of data, since no averages are required. However, it is worth noting that, in principle, an unbiased estimate of modal parameters can be obtained only by means of infinite records. Nevertheless, reliable estimates of natural frequencies are provided by SSI algorithms also in the case of records characterized by limited durations. Even if record length seems to be less critical for this class of methods, longer durations allow a more stable and reliable identification of modal parameters and, in particular, of damping ratios. In [32] a numerical study is reported, pointing out that at least 4000 data points are necessary for a reasonable identification of system frequencies lower than $1 \mathrm{~Hz}$ and damping ratios lower than $1 \%$.

\section{Literature damping data sets}

Since a model for structural damping analogous to those ones for mass or stiffness determination cannot be defined, the only alternative is to develop empirical expressions. In order to have reliable prior estimates of damping based on empirical formulations, a comprehensive database of experimental damping values is required. However, availability of damping data in the literature is not very large, in comparison with other data such as natural frequencies. Most of the available data are of limited value because variances are very large or not reported and because of the 
Table 1

Modal damping ratios: analysis results of data collected from the literature

\begin{tabular}{|c|c|c|c|c|c|c|c|c|c|c|c|c|c|}
\hline & & \multicolumn{12}{|c|}{ Type of construction } \\
\hline \multirow{2}{*}{\multicolumn{2}{|c|}{ Test conditions }} & \multicolumn{3}{|c|}{ Masonry bell towers } & \multicolumn{3}{|c|}{ Steel lattice towers } & \multicolumn{3}{|c|}{ Reinforced concrete buildings } & \multicolumn{3}{|c|}{$\overline{\text { Steel buildings }}$} \\
\hline & & $\begin{array}{c}\text { Mode } \\
\text { I } \\
\end{array}$ & $\begin{array}{c}\text { Mode } \\
\text { II }\end{array}$ & $\begin{array}{c}\text { Mode } \\
\text { III }\end{array}$ & $\begin{array}{c}\text { Mode } \\
\text { I } \\
\end{array}$ & $\begin{array}{c}\text { Mode } \\
\text { II }\end{array}$ & $\begin{array}{c}\text { Mode } \\
\text { III }\end{array}$ & $\begin{array}{c}\text { Mode } \\
\text { I } \\
\end{array}$ & $\begin{array}{c}\text { Mode } \\
\text { II }\end{array}$ & $\begin{array}{c}\text { Mode } \\
\text { III }\end{array}$ & $\begin{array}{c}\text { Mode } \\
\text { I }\end{array}$ & $\begin{array}{c}\text { Mode } \\
\text { II } \\
\end{array}$ & $\begin{array}{c}\text { Mode } \\
\text { III }\end{array}$ \\
\hline \multirow[t]{3}{*}{ Operational } & Av. $[\%]$ & 1.65 & 1.43 & 1.38 & 0.61 & 0.61 & 0.54 & 2.01 & 2.00 & 1.26 & 1.09 & 0.99 & 0.76 \\
\hline & St. Dev. [\%] & 0.77 & 0.89 & 0.53 & 0.42 & 0.35 & 0.30 & 1.36 & 0.97 & 0.66 & 0.49 & 0.46 & 0.43 \\
\hline & $\operatorname{COV}[\%]$ & 47 & 63 & 39 & 69 & 57 & 56 & 67 & 49 & 52 & 45 & 47 & 57 \\
\hline \multirow[t]{3}{*}{ Earthquake } & Av. $[\%]$ & N.A. & N.A. & N.A. & N.A. & N.A. & N.A. & 3.70 & 3.68 & $\begin{array}{c}\text { N.A. } \\
\text { (few data) }\end{array}$ & 2.18 & 2.10 & 1.95 \\
\hline & St. Dev. [\%] & N.A. & N.A. & N.A. & N.A. & N.A. & N.A. & 0.79 & 1.88 & $\begin{array}{c}\text { N.A. } \\
\text { (few data) }\end{array}$ & 1.13 & 0.53 & 0.14 \\
\hline & $\mathrm{COV}[\%]$ & N.A. & N.A. & N.A. & N.A. & N.A. & N.A. & 21 & 51 & $\begin{array}{c}\text { N.A. } \\
\text { (few data) }\end{array}$ & 52 & 25 & 7 \\
\hline
\end{tabular}

lack of descriptive data about the tested structure in terms, for example, of dimensions, characteristics of soil and foundations, architectural finishing and non-structural members, vibration amplitude. These characteristics, together with those ones of structural materials, test method and damping evaluation procedure contribute significantly to the dispersion of damping data. Moreover, it is worth emphasizing how short test durations or low quality data can result in large errors in damping estimation and, sometimes, in meaningless results: thus, such data cannot be included into a damping database aiming at the definition of empirical correlations. Preliminary results of analysis of damping data, collected during an extensive literature review carried out on this topic at the Structural and Geotechnical Dynamics Lab at University of Molise, are reported in Table 1 in terms of average values, standard deviation and coefficient of variation. No predictive formulations have been developed due to relative scarcity and high dispersion of available data, which at present make difficult identification of clear trends. They highlight the effect of amplitude of vibrations on damping (comparing operational and earthquake data), the effect of non-structural members on damping of steel structures (comparison between lattice tower and building data) and the higher damping values shown, on average, by reinforced concrete structures with respect to steel structures.

Experimental estimates of damping have been collected by many researchers over the years. Some of these collections are reported in [21] for tall buildings and [33,34] for bridges. According to this data sets, some empirical expressions for damping estimation have been proposed. For example, in [35] a simple formulation which correlates damping to the amplitude of vibration and to the building height is reported. A more complex model, taking into account building dimensions and its fundamental frequency, can be found in [21]. Base dimensions have been considered in this model to take into account the effects of radiation damping in the soil.

However, only recently some databases for damping values have been systematically organized. In particular, the Japanese Damping Database collects the dynamic properties of more than 200 steel and reinforced concrete buildings. They are mainly tall buildings (higher than $100 \mathrm{~m}$ ). An international database featuring the dynamic properties of 185 buildings in Asia, Europe and North America is, instead, described in [36]. Such databases include information about the buildings (location, usage, shape, height, dimensions, number of stories, structural type and foundation characteristics) and about dynamic properties (natural frequencies and damping ratios, together with information about excitation type, measurement method, data processing procedure and amplitude of vibrations).

Analysis of such data has given the following results:

- A dependency of damping from building height has been observed: in particular, it becomes smaller when the height increases. This is in agreement with the results reported in [37], where it is shown that soil-structure interaction leads to an increase in the overall damping of the system but it is less relevant for slender structures because of the effect of foundation rocking. Moreover, damping ratios much higher for reinforced concrete structures than for steel structures have been found.

- An increase of damping with the first natural frequency of the building has been also observed. Such a dependency is larger in the case of pile foundations: thus, radiation damping seems to give a high contribution to the overall damping for buildings supported with a pile foundation. This circumstance can be partially explained by the results of numerical analyses reported in [38], where it is shown that the system damping is influenced by the degree of contact between soil and lateral surface of the foundation. In particular, the smaller 
the structure slenderness and the shallower the soil layer, the more is the influence of the foundation depth and of the contact between soil and lateral surface of the foundation on system damping. Moreover, it significantly increases with layer depth, meaning that the damping capacity of the foundation for shallow layers is lower than for deep ones. The influence of soil-structure interaction and, therefore, of radiation damping on the overall damping of buildings is witnessed also by the higher scatter in damping values found for low-rise buildings (for which these effects are more relevant) with respect to high-rise ones.

- Non-structural members also cause an increase of structural damping.

A number of empirical expressions have been derived starting from these results. They basically correlate damping with the fundamental natural frequency and the height of the building. However, even if they allow a first evaluation of damping in a structure, the actual level of damping can be quite different because of the influence of other variables such as foundation type, soil conditions, quantity and arrangement of non-structural members. Moreover, due to the considerable scatter in the data, quality of correlations can be improved only by defining a standard and effective procedure for damping estimation.

\section{Optimized modal damping ratio estimation}

The actual level of damping for a given structural typology can be quite variable because of the influence of several factors, such as foundation type, soil conditions, quantity and arrangement of non-structural members, testing procedures and data processing methods. Thus, due to the considerable scatter in the data, quality of correlations can be improved first of all by defining a standard and effective procedure for damping estimation. When applying Operational Modal Analysis procedures for damping estimation, sensitivity analyses of modal parameters to record durations have pointed out the (sometimes relevant) bias introduced by short datasets. Here, some sensitivity analyses carried out in order to investigate the effect of the number of block rows on the estimated modal properties via SSI are reported.

The importance of evaluating the effect of the number of block rows on modal parameter estimates can be explained by reviewing the basic steps of SSI algorithms leading to modal identification.

Stochastic Subspace Identification methods work in time domain and are based on a state space description of the dynamic problem $[28,29]$. In fact, the second order problem, stated by the differential equation of motion, is converted into two first order problems, defined by the so-called "state equation" and "observation equation". Such equations, in the output-only case, can be written as follows:

$$
\begin{aligned}
& \left\{x_{k+1}\right\}=[A]\left\{x_{k}\right\}+\left\{w_{k}\right\} \\
& \left\{y_{k}\right\}=[C]\left\{x_{k}\right\}+\left\{v_{k}\right\}
\end{aligned}
$$

where $\left\{x_{k}\right\}=\{x(k \Delta t)\}$ is the discrete-time state vector yielding the sampled displacements and velocities, $\left\{y_{k}\right\}$ represents the sampled output, $[A]$ is the discrete state matrix, $[C]$ is the discrete output matrix, $\left\{w_{k}\right\}$ is the "process noise" due to disturbances and model inaccuracies, $\left\{v_{k}\right\}$ is the "measurement noise" due to sensor inaccuracy. These vector signals are both unmeasurable. They are assumed to be zero mean Gaussian white noise processes with covariance matrices given by:

$$
E\left[\left(\begin{array}{c}
\left\{w_{p}\right\} \\
\left\{v_{p}\right\}
\end{array}\right)\left(\left\{w_{q}^{T}\right\}\left\{v_{q}^{T}\right\}\right)\right]=\left(\begin{array}{c}
{[Q]} \\
{[S]} \\
{[S]^{T}[R]}
\end{array}\right) \delta_{p q}
$$

where $E$ is the expected value operator, $\delta_{p q}$ is the Kronecker delta (if $p=q$ then $\delta_{p q}=1$, otherwise $\delta_{p q}=0$ ), $p$ and $q$ are two arbitrary time instants. In the stochastic framework which characterizes OMA, due to the lack of information about the input, it is implicitly modeled by the noise terms $\left\{w_{k}\right\}$ and $\left\{v_{k}\right\}$. The white noise assumption about $\left\{w_{k}\right\}$ and $\left\{v_{k}\right\}$ cannot be omitted for the proof of this class of identification methods (see also [28]). If this assumption is violated, that is to say the input includes white noise and some additional dominant frequency components, such components will appear as poles of the state matrix $[\mathrm{A}]$ and cannot be separated from the eigenfrequencies of the system. Covariance Driven SSI (Cov-SSI) and Data Driven SSI (DD-SSI) are two algorithms for estimation of 
system matrices and, therefore, of eigenproperties. The main difference is related to the fact that Cov-SSI works on output correlations while DD-SSI works directly on raw data.

In the case of Cov-SSI, output correlations are computed as a first step:

$$
\left[\widehat{R}_{i}\right]=\frac{1}{N-i}[Y(1: N-i)][Y(i: N)]^{T}
$$

where $[Y(1: N-i)]$ is the data matrix with the last $i$ points removed, while $[Y(i: N)]$ is the data matrix with the first $i$ points removed; $N$ is the number of available data points. $\left[\widehat{R}_{i}\right]$ denotes that it is an estimate of the true correlation matrix at time lag $i$ based on a finite number of data: however, it is an unbiased estimate.

Correlation matrices are then gathered into a block Toeplitz matrix:

$$
\left[T_{1 \mid i}\right]=\left[\begin{array}{cccc}
{\left[\widehat{R}_{i}\right]} & {\left[\widehat{R}_{i-1}\right]} & \ldots & {\left[\widehat{R}_{1}\right]} \\
{\left[\widehat{R}_{i+1}\right]} & {\left[\widehat{R}_{i}\right]} & \ldots & {\left[\widehat{R}_{2}\right]} \\
\ldots & \ldots & \ldots & \ldots \\
{\left[\widehat{R}_{2 i-1}\right]} & {\left[\widehat{R}_{2 i-2}\right]} & \ldots & {\left[\widehat{R}_{i}\right]}
\end{array}\right]
$$

Each correlation matrix has dimensions $l \times l$, being $l$ the number of outputs. Thus, the block Toeplitz matrix has dimensions $l i \times l i$. If the system is of order $n$, in order to identify it the following condition must be fulfilled:

$$
l i \geqslant n
$$

The DD-SSI algorithm starts, instead, from a block Hankel matrix constructed directly from measurement data. It has $2 i$ block rows and $j$ columns (for the statistical prove of the method, it is assumed that $j \rightarrow \infty$, thus $j$ must be rather large). The value of $i$ is determined according to Eq. (12) as in the case of Cov-SSI. Said $l$ the number of outputs, the block Hankel matrix has dimensions $2 l i \times j$ and can be partitioned into the two sub-matrices of the past and future outputs as follows:

$$
\left[H_{0 \mid 2 i-1}\right]=\frac{1}{\sqrt{j}}\left[\begin{array}{cccc}
{\left[y_{0}\right]} & {\left[y_{1}\right]} & \ldots & {\left[y_{j-1}\right]} \\
{\left[y_{1}\right]} & {\left[y_{2}\right]} & \ldots & {\left[y_{j}\right]} \\
\ldots & \ldots & \ldots & \ldots \\
{\left[y_{i-1}\right]} & {\left[y_{i}\right]} & \ldots & \left.y_{i+j-2}\right] \\
\hline\left[y_{i}\right] & {\left[y_{i+1}\right]} & \ldots & {\left[y_{i+j-1}\right]} \\
{\left[y_{i+1}\right]} & {\left[y_{i+2}\right]} & \ldots & {\left[y_{i+j}\right]} \\
\ldots & \ldots & \ldots & \ldots \\
{\left[y_{2 i-1}\right]} & {\left[y_{2 i}\right]} & \ldots & {\left[y_{2 i+j-2}\right]}
\end{array}\right]=\left[\frac{\left[Y_{0 \mid i-1}\right]}{\left[Y_{i \mid 2 i-1}\right]}\right]=\left[\frac{\left[Y_{p}\right]}{\left[Y_{f}\right]}\right]
$$

These two sub-matrices have dimensions $l i \times j$. It is worth noting that output data are scaled by the factor $1 / \sqrt{j}$, in order to be consistent with the definition of covariance. In practical applications, the number of columns $j$ is taken equal to $N-2 i+1$, which implies that all given data samples are used. Another division is obtained by adding one block row to the past outputs and omitting the first block row of the future outputs:

$$
\left[H_{0 \mid 2 i-1}\right]=\left[\frac{\left[Y_{0 \mid i}\right]}{\left[Y_{i+1 \mid 2 i-1}\right]}\right]=\left[\frac{\left[Y_{p}^{+}\right]}{\left[Y_{f}^{-}\right]}\right]
$$

where, in this case, the superscript ${ }^{+}$denotes addition of one block row while in the following it denotes pseudoinverse.

The DD-SSI algorithm is based on the projection of the row space of the future outputs onto the row space of the past outputs. The definition of this projection (more details about projections can be found in [28]) is:

$$
\left[P_{i}\right]=\left[Y_{f}\right] /\left[Y_{p}\right]=\left[Y_{f}\right]\left[Y_{p}\right]^{T}\left(\left[Y_{p}\right]\left[Y_{p}\right]^{T}\right)^{+}\left[Y_{p}\right]
$$

From this definition it is clear that projections and covariances are closely related, being $\left[Y_{f}\right]\left[Y_{p}\right]^{T}$ and $\left[Y_{p}\right]\left[Y_{p}\right]^{T}$ block Toeplitz matrices containing covariances between the outputs. 
Table 2

Output-only modal identification results (EFDD)

\begin{tabular}{ccclcc}
\hline Record & $\begin{array}{c}\text { Number of } \\
\text { averages }\end{array}$ & Mode number & Type & $\begin{array}{c}\text { Natural } \\
\text { frequency }[\mathrm{Hz}]\end{array}$ & $\begin{array}{c}\text { Damping ratio } \\
{[\%]}\end{array}$ \\
\hline TdN1 & 42 & I & Translation & 0.813 & 0.75 \\
& & II & Translation & 1.375 & 1.09 \\
TdN2 & \multirow{2}{*}{69} & III & Torsion & 1.658 & 0.88 \\
& & I & Translation & 0.812 & 0.94 \\
& & II & Translation & 1.362 & 1.42 \\
& & III & Torsion & 1.627 & 1.21 \\
\hline
\end{tabular}

Table 3

Output-only modal identification results (Covariance-Driven SSI)

\begin{tabular}{ccclcc}
\hline Record & $\begin{array}{c}\text { Number of } \\
\text { block rows }\end{array}$ & Mode number & Type & $\begin{array}{c}\text { Natural } \\
\text { frequency [Hz] }\end{array}$ & $\begin{array}{c}\text { Damping ratio } \\
{[\%]}\end{array}$ \\
\hline TdN1 & 40 & I & Translation & 0.812 & 0.40 \\
& & II & Translation & 1.375 & 1.17 \\
TdN2 & \multirow{2}{*}{40} & III & Torsion & 1.657 & 0.63 \\
& & I & Translation & 0.812 & 0.68 \\
& & II & Translation & 1.362 & 1.31 \\
& & III & Torsion & 1.630 & 0.77 \\
\hline
\end{tabular}

The actual order of the system is obviously unknown but it can be estimated by looking at the peaks of the Power Spectral Densities (PSDs). After having estimated the order of the system, being the number of outputs a constant of the identification problem, the value of $i$ can be chosen. It is basically a user-choice based on a physical insight of the problem.

Then, if the system is observable and controllable, the rank of the block Toeplitz matrix or of the projection matrix equals the order $n$ of the system. The rank can be determined by applying SVD to the Toeplitz matrix or to the projection matrix. It is given by the number of non-zero singular values. This is a crucial step in SSI algorithms, since it allows to separate information from noise in the data before modal parameter extraction. In fact, only the non-zero singular values and the related singular vectors are used to obtain a realization of the $[A]$ and $[C]$ matrices and, therefore, to extract modal parameters from their eigenvalues and eigenvectors.

It is worth noting that, even if the order $n$ of the system can theoretically be obtained by inspecting the number of non-zero singular values, presence of noise leads to singular values all different from zero. Typical noise sources are modelling inaccuracies (for example, the system that generated the data cannot be modelled exactly as a stochastic state-space model), measurement noise (due to sensors and measurement hardware), computational noise (due to the finite precision of computers), the finite number of data points (as a consequence, the factorization property of the Toeplitz matrix or of the projection matrix does not hold exactly and their rank will not exactly be $n$ ). A rule of thumb suggests to look at the gap between two successive singular values. The singular value where the maximum gap occurs yields the model order. However, this criterion cannot be applied slavishly since actual structures often show no clear gaps. In order to find the modal properties of the system, therefore, it is better to construct a stabilization diagram. Thus, the order of the system is over-specified and structural modes are identified by looking for alignments of stable poles in order to distinguish physical modes from spurious ones.

The number of block rows, multiplied by the number of measurement channels, defines the maximum model order which can be selected during inspection of singular values of the projection or Toeplitz matrix. Even if the product of the number of block rows times the number of measurement channels is large enough to ensure that all modes in a certain frequency range can be identified, sensitivity analyses carried out on actual data point out that estimation of modal parameters and, in particular, of damping improves when the number of block rows increases, converging to a certain value [39]. Also the stabilization diagram becomes clearer. However, if the number of block rows is set much too high, spurious mathematical poles appear close to physical ones. They can be erroneously identified as stable but, above all, they bias damping estimates. Sensitivity analyses, carried out in order to investigate the influence of the number of block rows on the estimates, can therefore help to better define this parameter and to evaluate the level of uncertainty affecting the identified modal properties. 
Table 4

Output-only modal identification results (Data Driven SSI)

\begin{tabular}{ccclcc}
\hline Record & $\begin{array}{c}\text { Number of } \\
\text { block rows }\end{array}$ & Mode number & Type & $\begin{array}{c}\text { Natural } \\
\text { frequency [Hz] }\end{array}$ & $\begin{array}{c}\text { Damping ratio } \\
{[\%]}\end{array}$ \\
\hline TdN1 & 40 & I & Translation & 0.812 & 0.44 \\
& & II & Translation & 1.375 & 1.09 \\
TdN2 & \multirow{2}{*}{40} & III & Torsion & 1.656 & 0.59 \\
& & I & Translation & 0.812 & 0.74 \\
& & II & Translation & 1.361 & 1.19 \\
& & III & Torsion & 1.633 & 0.64 \\
\hline
\end{tabular}

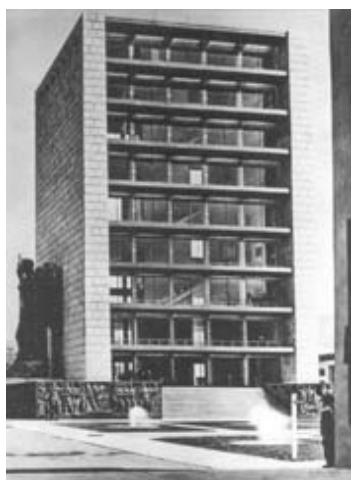

(a)

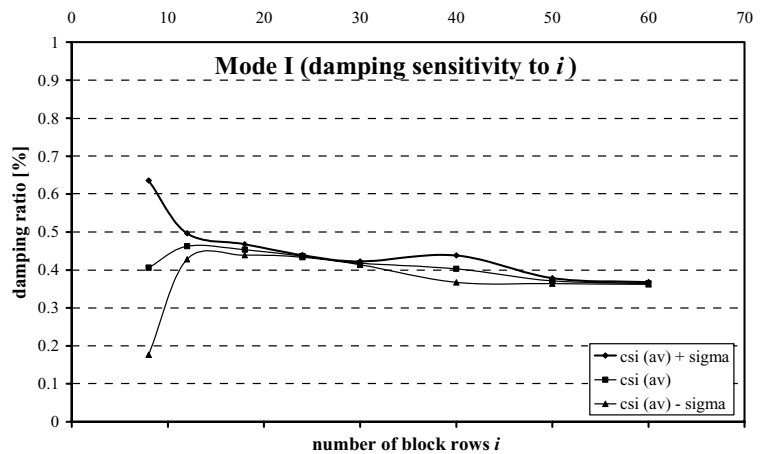

(b)

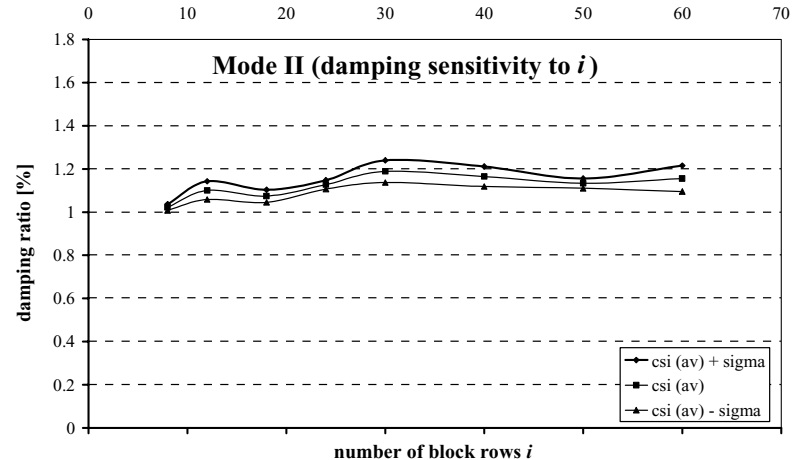

(c)

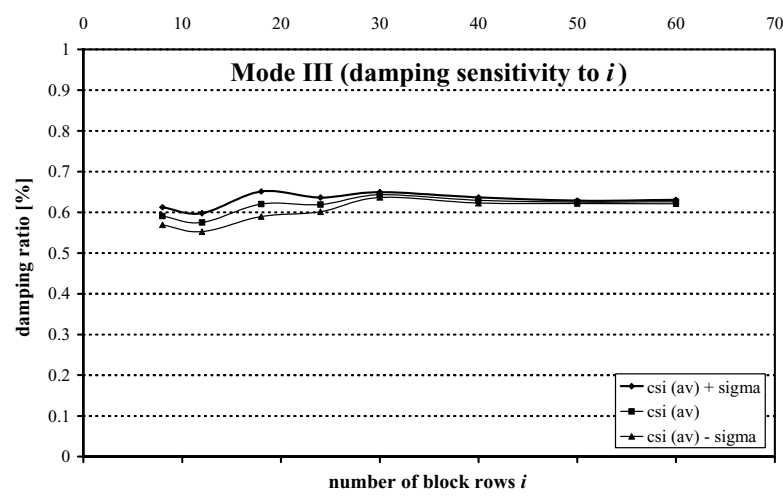

(d)

Fig. 1. a. The Tower of the Nations; b-c-d. Influence of the number of block rows on damping ratio estimates (TdN1 - Cov-SSI): "csi (av)" means average value of damping ratio, "sigma" its standard deviation.

In Tables 2, 3 and 4 the experimental estimates of modal damping ratios for a heritage reinforced concrete structure (the Tower of the Nations, Naples [39]) are reported. They have been obtained by applying the above described criteria for EFDD and SSI (both Covariance Driven and Data Driven). The structure is characterized by two seethrough and two completely blind (due to tuff masonry walls filling the reinforced concrete exterior frames) sides (Fig. 1a). Record length assured a high number of averages and a fine frequency resolution in the case of EFDD, and reliable estimates in the case of SSI. In Fig. 1b-c-d results of sensitivity analyses of damping ratio estimates with respect to the number of block rows are reported, allowing the identification of values of the number of block rows providing stable damping estimates, characterized by moderate variance. Estimates in good agreement each other have been obtained from the different methods. However, EFDD seems to provide a slight overestimation of damping ratio with respect to SSI methods. This is probably due to windowing, which is mitigated in presence of a high number of averages and a fine frequency resolution, but not completely removed. The influence of tuff masonry walls filling the reinforced concrete frames is pointed out by the higher value of damping ratio for the second mode 
in comparison with other modes. This circumstance can be probably addressed to a contribution of friction to the overall damping along the blind side.

\section{Conclusions}

The fundamental law of motion governing structural dynamics has been deeply investigated by many researchers, in particular to take into account the effect of damping on the dynamic behaviour of structures. In fact, damping strongly influences the structural response under dynamic loads but a single well established formulation to represent damping forces does not exist, yet. Since a model for structural damping analogous to those ones for mass or stiffness determination cannot be defined, an alternative is to develop empirical expressions. Nevertheless, recent developments in seismic design of structures point out the need of accurate a-priori estimates of damping, in particular when the Displacement-Based Design approach is adopted to prevent structural damages in presence of moderate earthquakes. Damping in structures can be then predicted by defining empirical correlations but this circumstance requires construction of a large database of consistent damping estimates for homogeneous classes of structures, reducing, at the same time, error bounds of experimental values of modal damping ratios.

Available damping data are generally scattered due to a number of different reasons: structural and soil characteristics, the need of defining a unique damping value (the so-called equivalent viscous damping) integrating different damping mechanisms, inherent limitations of data processing methods or inaccuracies in testing procedures (low quality data, short records). Thus, the main issues related to experimental estimation of damping have been reviewed in this paper.

Some criteria for a reliable experimental estimation of damping values have been identified, based on simulation results reported in the literature and sensitivity analyses carried out on actual measurements. A procedure for accurate damping estimation in operational conditions by SSI is also proposed and applied to a test case. Definition of testing protocols can reduce the influence of testing procedures on the scatter affecting damping estimates: this circumstance allows to focus attention on the actual sources of uncertainty. The present paper, therefore, aims at give a contribution towards standardization of testing and data processing procedures in order to keep error bounds in modal damping ratio estimates as low as possible.

Reduction of estimation errors allows construction of a large and consistent database, useful for definition of empirical expressions for a-priori estimation of damping values to be used in structural and seismic design. The extensive literature review and data collection carried out at the Structural and Geotechnical Dynamics Lab at University of Molise have underlined the variability of estimates and, above all, the scarcity of well reported relevant data. Whenever data about damping are provided, they may be not completely consistent also due to the lack of information about the tested structure and amplitude of vibration. Thus, the above mentioned damping database, aiming at providing reference values to be used in structural design, can result only from further work and definition of standardized dynamic test protocols.

\section{References}

[1] J. Woodhouse, Linear damping models for structural vibration, Journal of Sound and Vibration 215(3) (1998), 547-569.

[2] L. Gaul, The influence of damping on waves and vibrations, Mechanical Systems and Signal Processing 13 (1999), 1-30.

[3] G.D. Gounaris and N.K. Anifantis, Structural damping determination by finite element approach, Computers and Structures 73 (1999), $445-452$.

[4] L. Gaul and J. Becker, Damping Prediction of Structures with Bolted Joints, Proceedings of the International Conference on Structural Engineering Dynamics ICEDyn2009, Ericeira, Portugal, 2009.

[5] S. Lagomarsino, Forecast models for damping and vibration periods of buildings, Journal of Wind Engineering and Industrial Aerodynamics 48 (1993), 221-239.

[6] R.W. Clough and J. Penzien, Dynamics of Structures, McGraw-Hill, New York, USA, 1975.

[7] D.G. Fertis, Mechanical and Structural Vibrations, John Wiley \& Sons, New York, USA, 1995.

[8] M. Paz, Structural Dynamics: Theory and Computations, Chapman \& Hall, New York, USA, 1997.

[9] L. Gaul, S. Bohlen and S. Kempfle, Transient and forced oscillation of systems with constant hysteretic damping, Mechanical Research Communications 12 (1985), 187-201. 
[10] L. Gaul, Structural Damping in Frequency and Time Domain, Proceedings of the 7th International Modal Analysis Conference, Las Vegas, USA, 1989.

[11] J.T. Chen, On hysteretic damping mechanism in the time domain, NSC Report 85-2211-E-019-004, National Taiwan Ocean University, Taiwan, 1994.

[12] J.A. Inaudi and J.M. Kelly, Linear hysteretic damping and the Hilbert transform, Journal of Engineering Mechanics ASCE 121 (1995), 626-632.

[13] B.M. Fraejis de Veubeke, Influence of internal damping on aircraft resonance, AGARD Manual on Elasticity 1 (1960).

[14] T.K. Caughey and A. Vijayaraghavan, Free and forced oscillation of a dynamic system with 'linear hysteretic damping' (non-linear theory), International Journal of Non-Linear Mechanics 5 (1970), 533-555.

[15] S.H. Crandall, Dynamic Response of Systems with Structural Damping, Air, Space and Instruments, Draper Anniversary Volume, Edited by S. Lees, McGraw-Hill, New York, 1963, pp. 183-193.

[16] J.T. Chen and D.W. You, An integral-differential equation approach for the free vibration of a SDOF system with hysteretic damping, Advances in Engineering Software 30 (1999), 43-48.

[17] J.M.W. Brownjohn, Assessment of Structural Integrity by Dynamic Measurements, Ph.D. Thesis, University of Bristol, Bristol, England, 1988.

[18] Consiglio Superiore dei Lavori Pubblici, "Nuove Norme Tecniche per le Costruzioni”, D.M. Infrastrutture 14/01/2008, published on S.O. n. 30 at the G.U. 04/02/2008 n. 29, 2008.

[19] M.J.N. Priestley, G.M. Calvi and M.J. Kowalsky, Displacement-Based Seismic Design of Structures, IUSS Press, Pavia, Italy, 2007, ISBN: 8861980006

[20] ISO 4354:1997, "Wind Actions on Structures", International Organisation of Standardisation, 1997.

[21] A.P. Jeary, 'Damping in Tall Buildings - A Mechanism and a Predictor, Earthquake Engineering and Structural Dynamics 14 (1986), 733-750.

[22] A.P. Jeary, Damping in structures, Journal of Wind Engineering and Industrial Aerodynamics 72 (1997), 345-355.

[23] J.Q. Fang, A.P. Jeary, Q.S. Li and C.K. Wong, Random damping in buildings and its AR model, Journal of Wind Engineering and Industrial Aerodynamics 79 (1999), 159-167.

[24] D.J. Ewins, Modal Testing: Theory and Practice, Research Studies Press Ltd., Letchworth, Hertfordshire, UK, 1984.

[25] R. Brincker, C.E. Ventura and P. Andersen, Why Output-Only Modal Testing is a Desirable Tool for a Wide Range of Practical Applications, Proceedings of the 21st International Modal Analysis Conference (IMAC), Kissimmee, Florida, USA, 2003.

[26] R. Brincker, L. Zhang and P. Andersen, Modal Identification from Ambient Responses using Frequency Domain Decomposition, Proceedings of the 18th SEM International Modal Analysis Conference, San Antonio, TX, USA, 2000.

[27] S. Gade, N.B. Møller, H. Herlufsen and H. Konstantin-Hansen, Frequency Domain Techniques for Operational Modal Analysis, Proceedings of the 1st International Operational Modal Analysis Conference, R. Brincker and N. Møller, eds, Copenhagen, Denmark, 2005.

[28] P. Van Overschee and B. De Moor, Subspace Identification for Linear Systems: Theory - Implementation - Applications, Dordrecht, the Netherlands: Kluwer Academic Publishers, 1996.

[29] B. Peeters, System Identification and Damage Detection in Civil Engineering, Ph.D. Thesis. Katholieke Universiteit Leuven, Leuven, Belgium, 2000.

[30] J.S. Bendat and A.G. Piersol, Random Data: Analysis and Measurement Procedures, John Wiley \& Sons, New York, USA, 1986.

[31] Y. Tamura, A. Yoshida, L. Zhang, T. Ito, S. Nakata and K. Sato, Examples of Modal Identification of Structures in Japan by FDD and MRD Techniques, Proceedings of the 1st International Operational Modal Analysis Conference, Copenhagen, Denmark, 2005.

[32] B.A. Pridham and J.C. Wilson, A study on errors in correlation-driven stochastic realization using short data sets, Probabilistic Engineering Mechanics 18 (2003), 61-77.

[33] A.G. Davenport, Reliability of Long Span Bridges Under Wind Loading, Proceedings of ICOSSAR, Trondheim, Norway, 1981.

[34] R. Eyre and G.P. Tilly, Damping Measurements on Steel and Composite Bridges, Symposium on dynamic behaviour of bridges, Crowthorne, Berks, USA, 1977.

[35] A.G. Davenport and P. Hill-Caroll, Damping in Tall Buildings: its Variability and Treatment in Design, ASCE spring convention, Seattle, USA, 1986.

[36] S. Lagomarsino and L.C. Pagnini, Criteria for modelling and predicting dynamic parameters of buildings, Report ISC-II, 1, Istituto di Scienza delle Costruzioni, University of Genoa, Genoa, Italy, 1995.

[37] A.S. Veletsos and J.W. Meek, Dynamic behaviour of building-foundation systems, Earthquake Engineering and Structural Dynamics 3 (1974), 121-138.

[38] J. Avilés and L.E. Pérez-Rocha, Evaluation of interaction effects on the system period and the system damping due to foundation embedment and layer depth, Soil Dynamics and Earthquake Engineering 15 (1996), 11-27.

[39] C. Rainieri, Operational Modal Analysis for Seismic Protection of Structures, Ph.D. Thesis, University of Naples "Federico II", Naples, Italy, 2008. 

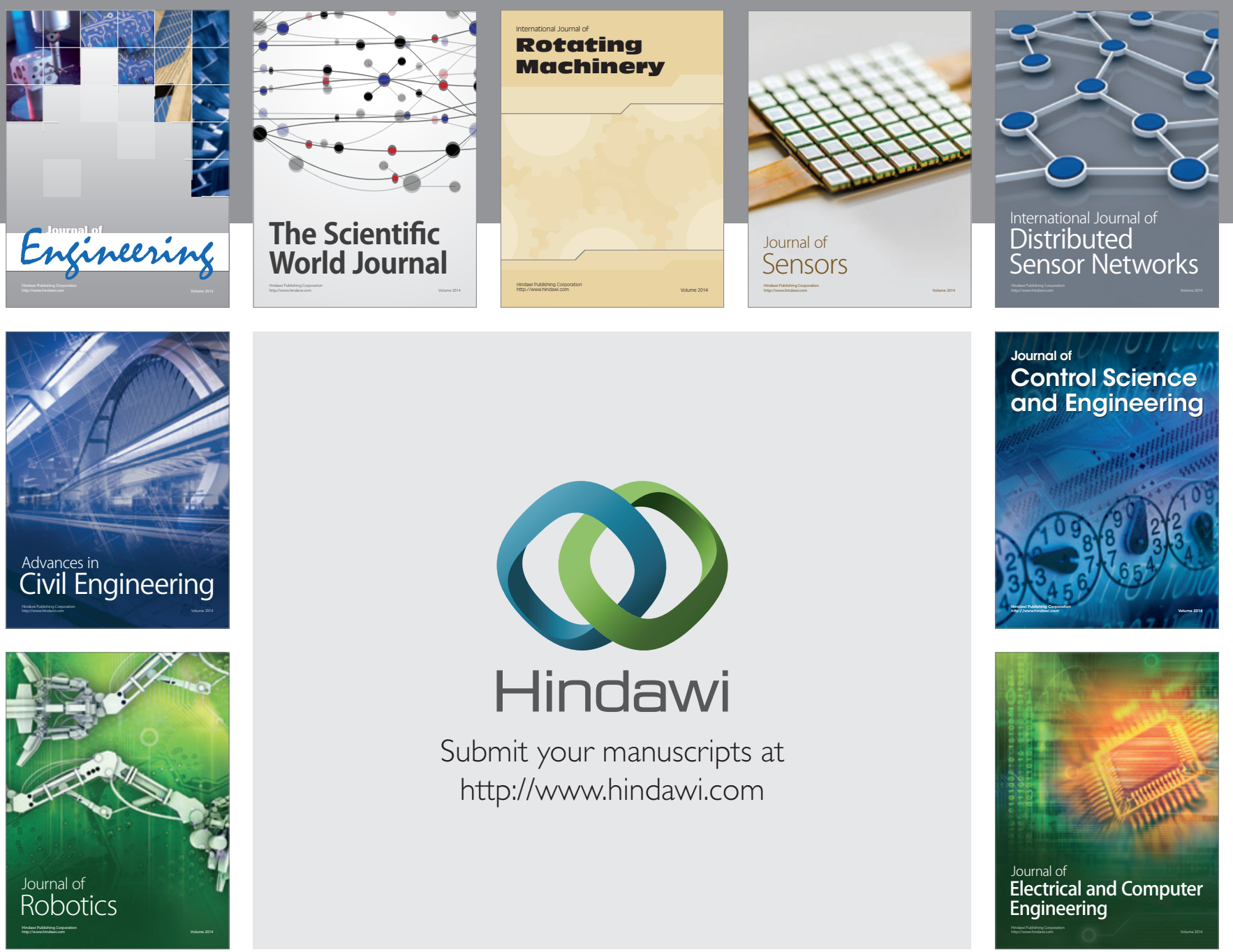

Submit your manuscripts at

http://www.hindawi.com
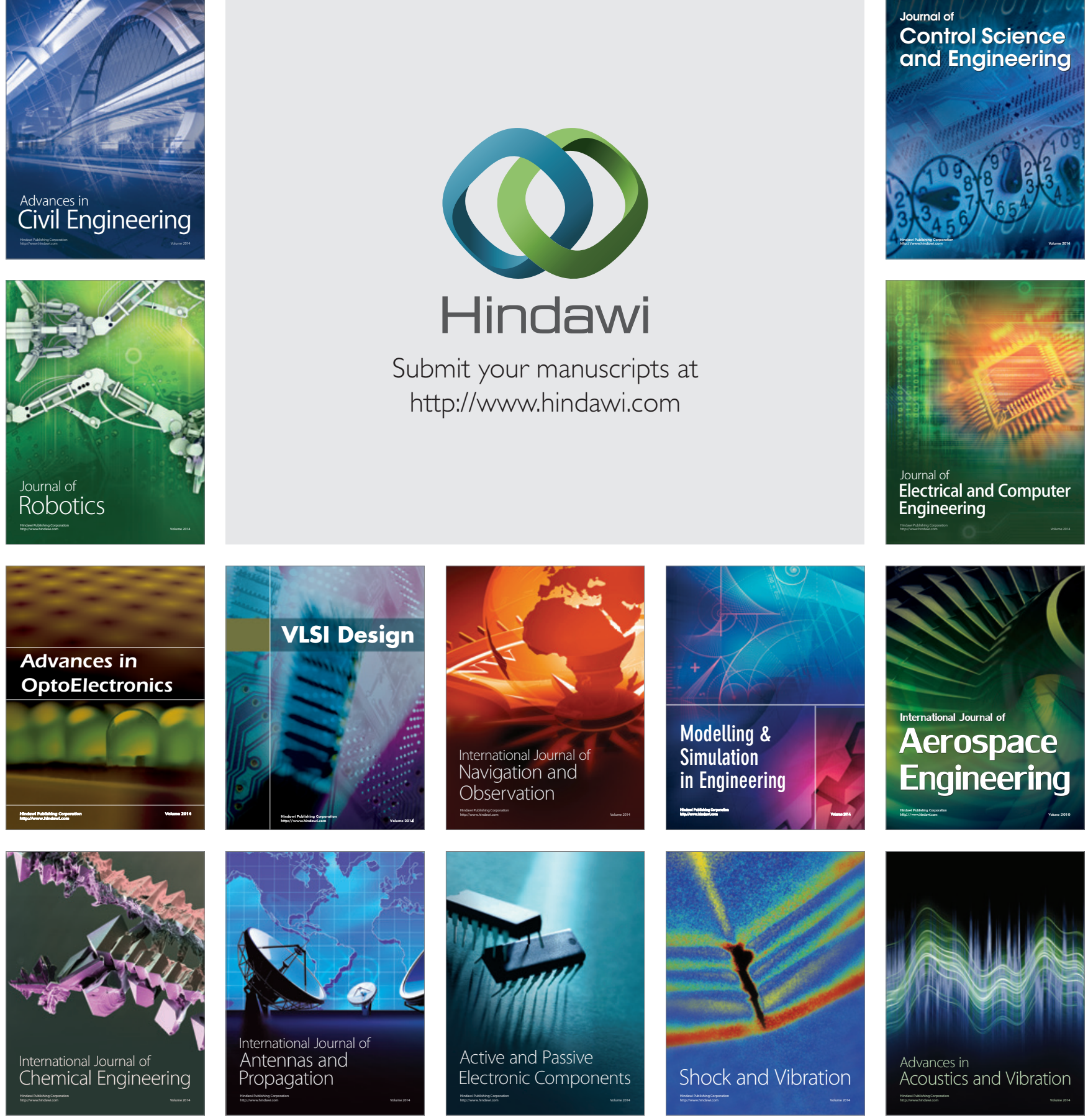